\title{
Questions autour des neurosciences et de leur pouvoir explicatif
}

Jean Martin

jean.martin[at]saez.ch
Un article récent du magazine The New Yorker, par Adam Gopnik [1], a attiré mon attention. Sur un sujet scientifique qui pose de nombreuses questions au plan sociétal et notamment éthique, cette contribution m'a intéressé au vu du caractère généraliste de haut niveau et (intelligemment) critique de la revue hebdomadaire en question - qui est de celles qui comptent aux Etats-Unis, par la qualité de ses textes et de ses journalistes - traitant de thèmes politiques et économiques aussi bien que culturels, littéraires et scientifiques (les dessins humoristiques qui parsèment ses pages sont aussi fameux). Extraits ci-dessous.

\section{Neuro-enthousiastes et neuro-sceptiques}

«Les auteurs traitant du cerveau et de l'esprit se divisent entre ceux qui admettent un moi rationnel et analytique et que ce qu'il fait dépend de mécanismes logiques, et ceux qui croient que ce qui gouverne notre vie est non seulement irrationnel mais encore inexplicable, et que c'est bien ainsi. Depuis plus de dix ans, les rationnels tenaient le haut du panier (...) le «virage neurologique» devenant ce que le «virage culturel» était il y a quelques décennies: l'explication de tout et à toutes fins utiles. A l'époque, le magazine Time rendait hommage, à propos de pour-

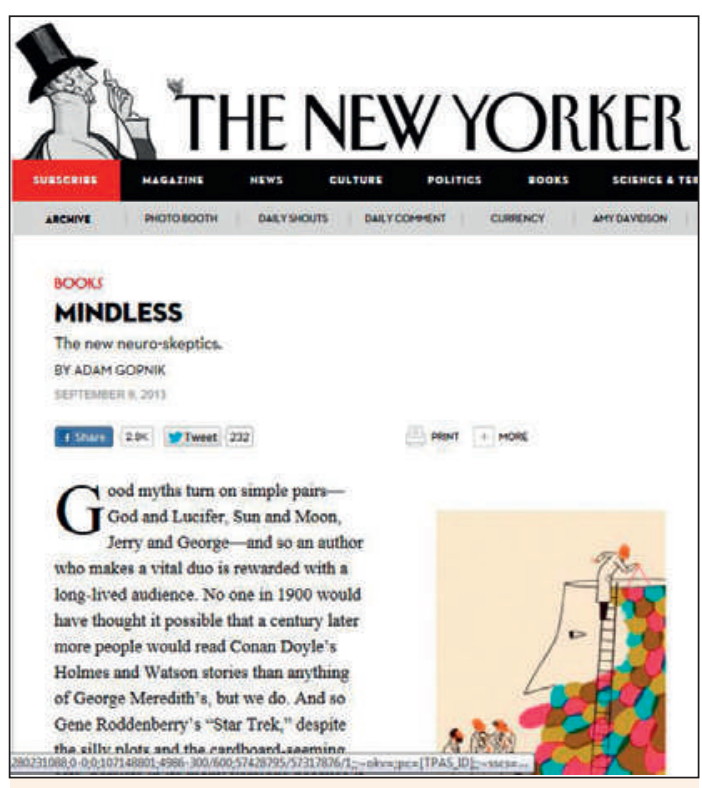

On peut lire la version online du texte d'A. Gopnik sous: www.newyorker.com/arts/critics/books/2013/09/09/ 130909crbo_books_gopnik?currentPage=all suite du plaisir, au livre de Christopher Lasch «The culture of narcissism»; aujourd'hui, le même journal a un article majeur sur le bonheur en se demandant «dans quelle mesure nous sommes connectés organiquement (hardwired) pour le rechercher». Gopnik relève que cette division a pris une nouvelle vigueur: nous donnons d'autant plus d'attention à la personne comme machine pensante au moment où nous commençons à avoir des machines qui pensent!

Sont évoqués des livres récents présentant des arguments tendant à démontrer que les neurosciences «promettent beaucoup mais apportent peu». En particulier «A skeptic's guide to the mind», par Robert A. Burton (St. Martin's Press), «Brainwashed: the seductive appeal of mindless neuro-science», par Sally Satel et Scott O. Lilienfeld (Basic Books), et «Neuro: the new brain sciences and the management of the mind», par Nikolas Rose et Joelle M. Abi-Rached (Princeton Univ. Press).

\section{Organicité «topographique» et/ou plasticité fonctionnelle}

Gopnik compare certains des efforts d'interprétation actuels dans les neurosciences à la phrénologie de Franz Joseph Gall, qui a eu ses heures de gloire au $\mathrm{XIX}^{\mathrm{e}}$ siècle (surtout dans sa première moitié), qu'il caricature comme «bumpology» (sciences des bosses... du crâne ou du cerveau). Ce qui est clair, et apparaît dans les ouvrages qu'il discute, c'est qu'il est très rare qu'une activité mentale quelconque soit située de manière précise dans un réseau spécifique de neurones ou un «morceau» du cerveau. «Tous les neurosceptiques insistent sur la plasticité de nos réseaux neuronaux (...) Nous apprenons et formatons notre status neurologique autant que nous l'héritons». «Ourselves shape our brains at least as much as our brains shape our selves» (il me semble toutefois - note de J.M. - que tous les neuroscientifiques, y compris les enthousiastes, peuvent se dire d'accord avec cela).

\section{Préoccupation des neuro-sceptiques: la mise en cause du libre arbitre}

«Chaque auteur cependant propose un projet polémique pour remplacer les modèles catalogués comme ‘phrénologiques». Le livre Brainwashed est vivement libertarien; une des craintes de ses auteurs est que la neuroscience fragilise et déplace la notion de responsabilité individuelle pour une mauvaise ac- 
tion (délit, crime) en l'attribuant à un irresponsable cerveau, permettant à des malfaiteurs de prendre à témoin la science pour ne pas être condamnés». Selon Gopnik, le neurologue Burton, auteur de A skeptic's guide, se mue en philosophe et veut démontrer que la neuroscience ne peut rien dire sur le libre arbitre et le déterminisme, et qu'elle ne pourra jamais le faire. Pour Burton, «L'esprit est et sera toujours un mystère». Examiner avec nos esprits le fonctionnement de nos esprits serait comparable à écrire un livre sur les hallucinations alors qu'on est sous LSD...

\section{Les observations (les mises en évidence) des neurosciences ne nous apprendraient que peu} Les auteurs du livre Neuro mettent le doigt sur un point: à leur avis la neuroscience est souvent capable de répondre aux questions évidentes mais rarement à celles qui sont intéressantes. «Elle peut nous dire comment nos esprits sont constitués pour entendre de la musique mais pas pourquoi Mozart est plus profond que d'autres». Ils prennent à partie les expériences connues de Benjamin Libet qui semblent discréditer la notion de libre arbitre - Libet a montré que, déclenchant un mouvement musculaire, le cerveau commande, choisit, quelques millisecondes avant que le sujet ne réalise qu'un choix a été fait. Rose et Abi-Rached sont très sceptiques quant à l'idée que ces observations nous apprennent quoi que ce soit sur l'exercice de la volonté humaine dans la vie quotidienne. La signification de ce qu'est le libre arbitre comme pratique sociale humaine ne peut simplement pas être déduite d'une mesure neurologique.

Ainsi, une objection de ces auteurs est que les explications apportées par les neurosciences ne font que redire ce qu'on sait sans elles. Affirmer qu'une émotion est réelle parce que, d'une manière ou de l'autre, on peut la voir se produire par des techniques sophistiquées n'ajoute rien à la compréhension qu'on peut en avoir. Satel et Lilienfeld parlent de «neuro-redondance» à propos de ces choses que nous savions déjà avant le scan du cerveau et autres moyens. Et se moquent d'un chercheur qui affirme que le syndrome de stress post-traumatique est une vraie maladie parce que l'imagerie cérébrale permet de mettre en évidence certains signes. C'est là certainement (note de J.M.) une remarque qui mérite considération! On pense au fait qu'une bonne partie de l'activité des médecins de famille, parmi d'autres, illustre que les signes objectivables ne sont pas les seuls qui comptent.

\section{Les neurosciences contribuent au débat entre nature et culture}

Il reste que malgré les critiques qu'ils émettent, dit Gopnik, même les neuro-sceptiques acceptent que les avancées actuelles des neurosciences représentent un correctif important à un certain anti-organicisme radical, aux théories selon quoi le cerveau (vu en termes physiques), n'a pas d'importance et que la culture construit tout. Dans la foulée des connaissances anatomiques et physiologiques antérieures, les neurosciences confirment de manière plus précise des données solides. Prenant un exemple simple: une tumeur ou un hématome exerçant une pression sur une zone du cerveau a des conséquences, potentiellement graves, entraînant des troubles psychologiques aussi bien que physiques. «Le fait est que les questions et manifestations de culpabilité, de tentation ou de tabou, dans le registre freudien ou, beaucoup plus tôt, celui de Sophocle, peuvent être accentuées ou transformées par une pression physique dans le cerveau. On ne peut que respecter le pouvoir de la 〈viande〉 (sic) de changer aussi clairement des éléments moraux».

\section{Neurosciences et humanisme}

Comme d'habitude pourrait-on dire, la réalité se trouve quelque part entre - ou à la fois dans - les convictions exprimées par les neuro-sceptiques et celles de ceux qui croiraient exclusivement aux aspects démontrables par la technique. C'est ce que discute Patricia S. Churchland dans son ouvrage «Touching a nerve: the self as brain» (Norton), où elle présente une défense de l'importance de la neurologie scientifique. En critiquant vivement ceux qui traitent péjorativement de «scientisme» les apports de la neuroscience à la philosophie, par exemple, comparant cette résistance à celle qu'a rencontrée à l'époque Galilée, ou Harvey à propos de circulation du sang.

Forte formulation: «En réalité, l'humanisme n'a pas seulement survécu aux démystifications successives - que nous devons à Galilée, Harvey ou d'autres, il a été renforcé par la démonstration du pouvoir d'explication de l'investigation rationnelle dont dépend l'humanisme lui-même. La volonté de diminuer la part de mystère dans la nature, s'efforcer de nommer, comprendre, les lois naturelles que nous n'avons pas nous-mêmes élaborées, ce n'est pas du scientisme, c'est de la science». Et Churchland de souligner combien le travail du cerveau est contingent, comment il peut être simple («mécanique») à certains égards, «monstrueusement complexe» à d'autres.

Et en guise de conclusion: «La neuroscience ne peut pas nous enlever la responsabilité de nos actions mais elle peut nous soulager de la culpabilité de n'être que simplement humain. Nous serons moins mal dans nos dépressions et autres «mental breakdowns» si nous comprenons les pannes du cerveau qui en sont les causes ou les favorisent».

1 Gopnik A. Mindless - The new neuro-skeptics. The NewYorker. September 9. 2013; p. 86-8. www.newyorker.com/arts/critics/ books/2013/09/09/130909crbo_books_ gopnik?currentPage $=$ all 